months it is too early to predict their ultimate outcome. So far two children, one of whom required a shunt for post-haemorrhagic hydrocephalus, show signs of moderate developmental delay.

\section{Discussion}

As intensive care places considerable emotional strain on both parents and nursing staff, it is perhaps wise to question whether those babies with a particularly poor prognosis should be treated. Separation from the mother in these circumstances, especially if referral to a regional neonatal unit is necessary, is undesirable and may seriously interfere with grieving.

Over the past six months many such parents have written asking for either a photograph (easily forgotten when caring for critically ill babies) or at least some memento of the birth (for example a name band). After discussion with the parents, withholding long term intensive care may in certain circumstances be the most appropriate course of action.

We have found that bruising is almost always a fatal sign in these babies. Only one of $18(5.6 \%)$ bruised babies (mean birthweight $755 \mathrm{~g}$ ) survived compared with 9 of 12 without bruising (mean birthweight $755 \mathrm{~g}$ ). This may be because bruising is often associated with an intraventricular haemor- rhage. This was true for $59 \%$ of our babies who had a necropsy, and similar findings have been reported by others. ${ }^{3}$ The aetiology of bruising is unclear; it may be due to a degree of birth asphyxia or trauma, or may simply be a sign of the extreme fragility of blood vessels. Delivery by caesarean section has been advocated ${ }^{4}$ for very preterm infants in order to prevent complications, but 11 of our 18 bruised babies were delivered in this way.

As a result of these findings, although we resuscitate all babies born in our unit, we would no longer routinely undertake long term respiratory support in infants with skin bruising and fused eyelids.

\section{References}

1 Campbell AGM. Which infants should not receive intensive care. Arch Dis Child 1982;57:569-71.

2 Hirata T, Epear JT, Walsh A, et al. Survival and outcome of infants 501 to $750 \mathrm{gm}$ : a six year experience. J Pediatr 1983;102:741-8.

${ }^{3}$ Szymonowicz W, Yu VYH, Wilson FE. Antecedents of periventricular haemorrhage in infants weighing $1250 \mathrm{~g}$ or less at birth. Arch Dis Child 1984;59:13-7.

${ }^{4}$ Haesslein HC, Goodlin RC. Delivery of the tiny newborn. Am J Obstet Gynecol 1979;134:192-8.

Correspondence to Dr P Congdon, Clarendon Wing, General Infirmary at Leeds, Belmont Grove, Leeds LS2 9NS.

Received 14 December 1985

\title{
Campylobacter enteritis and bloody stools in the neonate
}

\author{
E R YOUNGS, C ROBERTS, AND D C DAVIDSON
}

\section{Public Health Laboratory and Maternity Unit, Fazakerley Hospital, Liverpool}

SUMMARY Within 72 hours of birth three babies had loose stools containing fresh blood, mucus, and Campylobacter jejuni/coli. Campylobacter enteritis should be considered in newborn babies passing blood per rectum.

Campylobacter jejuni and $C$ coli are prevalent causes of acute bacterial gastroenteritis in humans. From available figures, however, the incidence of infection during the neonatal period is probably low. For example during 1977-80, of 2255 cases reported to the Communicable Diseases Surveillance Centre for whom age details were available, $57(2.5 \%)$ were neonates. Consequently, in Great Britain the clinical features, including complications of acute infection, are well recognised in older patients but are less well documented in the newborn. Mawer and Smith ${ }^{1}$ described a baby of 34 weeks' gestation with campylobacter in his stools who did not manifest signs of infection possibly because of previous antibiotic treatment.

We describe three newborns with bloody stools from which $C$ jejuni/coli (one case) and $C$ jejuni (two cases) were isolated.

\section{Case reports}

Patient 1. A girl weighing $3.5 \mathrm{~kg}$ was born by normal delivery at term 30 minutes after membrane rupture. This followed several days of maternal nausea, abdominal pain, loose stools, and mild pyrexia which was subsequently diagnosed as campylobacter enteritis. On the third day after birth the baby passed a loose stool containing fresh blood and mucus and yielding $C$ jejuni/coli (this organism was not further identified), and in the subsequent 24 hours passed two more similar stools before they 
returned to normal. Oral erythromycin $(60 \mathrm{mg}$ tds) was given for seven days during which time she remained well.

Patient 2. A girl of 36 weeks' gestation weighing $2 \cdot 6$ $\mathrm{kg}$ was born by assisted breech delivery 10 minutes after rupture of the membranes. The same day her mother had complained of diarrhoea and $C$ jejuni was isolated from her stool. Forty hours after delivery the baby passed a green loose stool mixed with blood and mucus which recurred in the following 24 hours. Culture yielded $C$ jejuni and erythromycin ( $31.25 \mathrm{mg}$ qds) was given. A small anal fissure was noted but the baby remained well and stool culture was negative within four days.

Patient 3. A girl weighing $3.6 \mathrm{~kg}$ was born at term by normal delivery 10 minutes after membrane rupture. A day later her mother developed a punctate rash over her limbs but was otherwise well. Three days after birth the baby passed three watery stools mixed with blood and mucus. The rectal mucosa was red and friable but the stools returned to normal within 72 hours. $C$ jejuni was recovered from both mother and baby, although the former had had no recent gastrointestinal disturbance. The baby was not given erythromycin and remained well but still excreted the organism 10 days later.

\section{Discussion}

These three case histories illustrate the relatively benign nature of $C$ jejuni/coli infection in newborns, albeit with supportive hospital measures including erythromycin in two babies. None of these babies had true gastroenteritis. One had frankly watery stools; the other two had loose but not watery stools. All three passed fresh blood and mucus. This is in general agreement with reports from other countries in neonates,$^{2-5}$ in whom signs were generally confined to the gastrointestinal tract. Another report, however, illustrates this organism's potential to infect beyond the intestine. ${ }^{6} C$ jejuni/coli was isolated from cerebrospinal fluid of a 12 day old baby. There was no history of gastrointestinal disturbance and recovery followed treatment which included chloramphenicol and gentamicin. This case is more characteristic of infection with a taxonomically related organism Campylobacter fetus which usually presents in the first three weeks of life with septicaemia and meningitis, but is infrequently reported.

Although the three mothers here had $C$ jejuni/coli in their stools, vaginal swabs were not cultured specifically for these organisms. They are not part of the normal vaginal flora but one baby's mother reported by Anders $e t \mathrm{al}^{4}$ had the organism in the vagina. It may be possible therefore that ingestion of $C$ jejuni/coli (from vaginal as well as faecal origin) by a baby could occur during vaginal delivery.

Patient 2 could have had bleeding associated with her anal fissure, but fresh blood loss per rectum in a newborn should prompt clinical awareness and microbiological investigation for $C$ jejuni/coli in addition to other disorders including necrotising enterocolitis. (In our experience anal fissures are not infrequently associated with necrotising enterocolitis and other forms of colitis in the newborn). The differential diagnosis is more apparent if there is maternal diarrhoea but as in patient 3 this is not always present either before or after delivery. Infection in the neonatal period generally seems to cause a much milder gastrointestinal disturbance than that in children and adults. Whether erythromycin treatment is needed in neonatal infections is debatable and a controlled trial is unlikely to be carried out because of the small numbers involved. Comparison of patients 1 and 2, however, with patient 3 would suggest that erythromycin can curtail carriage of $C$ jejuni/coli in the neonate. Even in patient 3 the illness was self limiting without antimicrobial treatment (as in adults) since stools returned to normal within three days. Therefore, it would seem that the signs of campylobacter enteritis were not materially affected by the administration of erythromycin. Buck et al $l^{5}$ reported that loose stools continued for three and five days respectively in their neonates, and macroscopic blood staining ceased after one and two days respectively while both were receiving erythromycin.

It would be prudent for clinicians and microbiologists to consider $C$ jejuni/coli in association with bloody stools in the newborn. Furthermore the need for antimicrobial cover to curtail carriage and possible invasion in the neonate, as well as preventing spread within the unit should be borne in mind.

\section{References}

${ }^{1}$ Mawer SL, Smith BAM. Campylobacter infertion of premature baby. Lancet 1979;i:1041.

2 Karmali MA, Tan YC. Neonatal campylobacter enteritis. Can Med Assoc J 1980;122:192-3.

3 Vesikari T, Huttunen L, Mäki R. Perinatal Campylobacter fetus ss jejuni enteritis. Acta Paediatr Scand 1981;70:261-3.

4 Anders BJ, Lauer BA, Paisley JW. Campylobacter gastroenteritis in neonates. Am J Dis Child 1981;135:900-2.

${ }^{5}$ Buck GE, Kelly MT, Pichanick AM, Pollard TG. Campylobacter jejuni in newborns: a cause of asymptomatic bloody diarrhoea. Am J Dis Child 1982;136:744.

6 Thomas K, Chan KN, Ribeiro CD. Campylobacter jejuni/coli meningitis in a neonate. $B r$ Med $J$ 1980;280:1301-2.

Correspondence to Dr E R Youngs, Public Health Laboratory, Queen's Medical Centre, Nottingham NG7 2UH.

Received 7 January 1985 